\title{
Improved Computer Network Protocol for Secondary Users' Informatioin Exchanges in a Cognitive Radio
}

\author{
Haiming $\mathrm{Xu}^{1, \mathrm{a}}$, Weijie $\mathrm{Wu}^{1}$, Tian Zhang $^{1}$, Donglin Wang ${ }^{2, \mathrm{~b}}$ \\ 1.EE Department, Nanjing Univ. of Posts and Telecommun. \\ ECE Department, New York Institute of Tech. at Nanjing,Nanjing, China \\ azjxhmxnh@163.com \\ 2.Department of Electrical and Computer Engineering \\ New York Institute of Technology at Nanjing Campus,Nanjing, China \\ bdwang09@nyit.edu
}

\begin{abstract}
Keywords:Cognitive Radio; Energy detection; Computer Network protocol; Multiple SUs'
\end{abstract}
\begin{abstract}
With the development of the communication network, the cognitive radio becomes good enough to provide the reliable spectrum resources and reliable communications for the secondary users (SUs).This paper considers improving a computer network protocol and then utilizing this computer protocol for secondary users' (SUs') information exchanges in cognitive radio. Energy detection is used to find the idle spectrum resource of the primary user (PU).The threshold value can be found outto guarantee that the energy detector can run in the optimal performance. Thus, the physical layer can be built in a good condition. Then the computer network protocol, i.e. UPD, can be utilized for information exchanges between SUs for better spectrum sensing. Simulation results are provided to demonstrate our proposed method.
\end{abstract}

\section{Introduction}

The computer network has faced continuous development in recent years and more and more theory has been put into application to meet the higher and higher demand for network performance, especially the high-required demand for the network's ability to support mobile computing. The definition about mobile computing is the performance that the network terminal are able to be roaming in the network and maintain network communication at the same time. However, in the traditional situation, large area network (LAN) cannot meet the requirement; in this case, the wireless LAN technology was introduced and appeared rapidly development.

After a period of time, the people have experience the great development of the wireless technology. Many applications about the wireless technology have great improved the social efficiency and the quality of human life in civilian field. It can meet the communication needs of field activities and disaster relief action, which can be used to combat units, elementary relief systems and the communication systems between urban vehicles. It also can be used to satisfy the demand that some temporary communication network can be built quickly. Under this background, the shortage of the spectrum resource imposes a big restriction on the application.

Although the demand of spectrum has greatly increased, owing to the rapid development of wireless network, the spectrum resource which can be used is limited. The data shows that the available resource cannot support such amount of wireless devices. Fortunately, according to , a large proportion of licensed spectra cannot be made fully use of today, which means people still have the chance to develop and improve the wireless technology. Therefore, cognitive radio (CR) [2-3] and dynamic spectrum access (DSA) [4] were proposed by the scientists and have been experienced further development. In this field, the licensed users are called primary users (PUs) [5]. The study shows that allowing the licensed spectrum to be shared by secondary users (SUs) can improve remarkably the spectrum efficiency. For this context, the new concept - SUs [6] are proposed and play an important role in the application. The technology of detection, like energy detection [7] and cooperative spectrumsensing [8], appears to support the study. 
Most of the protocol is based on the characteristics of the wireless situation-limit of power, instability and so on. However, nowadays the new technique can overcome mast of the shortcoming, which means wireless network can be considered as a wire network in some situation. This paper is based on this idea and use the common computer network protocol to build ad hoc network, with the help of UDP [9] and CBR [10-11].

The paper is organized as followings: Section IIistalking about the cogitative radio technology, i.e. spectrum sensing and energy detection and the threshold will be found to guarantee that the detector can run in optimal performance; Section III offers details about UDP and CBR; Section IV offersthe steps about the construction of the ad hoc network and includes the results of the simulation. The last section is the conclusion about the research.

\section{Spectrum Sensing}

\section{Energy detection.}

The best sensing method is the energy detection, when SUs cannot get enough information of the PU's signal. This is a simple spectrum sensing method, whose verdict doesn't need to be based on the prior information. In this case, the energy detector will never require a prior information about the PU's signals, but the detectors need to know the power of the white Gaussian noise in the background. Figure 1 shows the flowchart of energy detection algorithm.

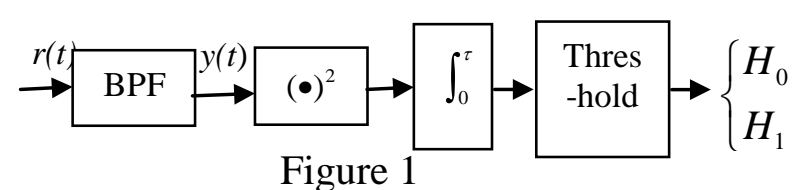

From the flow chart above, it shows that SUs can get the verdict based on the threshold, by calculating the energy of the received signal which is received by band pass filter.

\section{Energy Detection Model.}

Cognitive radio system makes the verdict that whether band is used by detecting the signals from the PU and looking for spectrum hole. According to the statement above, the detection problem can be solved as the following model:

$$
y(n)=\left\{\begin{array}{ll}
v(n) & \text { for } H_{0} \\
p(n)+v(n) & \text { for } H_{1}
\end{array}, 0 \leq n \leq N-1,\right.
$$

In Eq.(1), $y(n)$ is defined as the received signal at $\mathrm{SU} ; \mathrm{N}$ is thenumber of samples; $p(n)$ is defined as an unknown PU signal which suffers from the condition of the wireless channel, like attenuation, loss, delay and so on; and $v(n)$ is an Additive White Gauss Noise (AWGN) with zero mean and variance of $\sigma^{2}$.

In this model, the Hypothesis $H_{0}$ is defined that PU is inactive, while hypothesis $H_{1}$ is defined that PU is active. The energy detection will make the verdict by detecting the signal energy and comparing it with value of the preset threshold.

\section{Probability of Detection And Calculation of Threshold.}

In this section, the premise that $p(n)$ and $v(n)$ are independent on each other and the average power of the signal is the only information for the energy detection system should be made to validate the following solution procedure.

At first, a test statistic needs to be defined to assist the system make the verdict. The test statistic for energy detection can be expressed as:

$$
T(y)=\frac{1}{W} \sum_{n=0}^{W-1}|y(n)|^{2},
$$

In Eq.(2), $y(n)$ is the signal as given in Eq.(1),which is sensed by SU and $\mathrm{W}$ indicates the length of the sensing window, whose meaning is its discretelength in samples. 
On the condition that the number of samples in the signal $\mathrm{N}$, if the noise average power is constant, sensing signals transmitted from PU user receives can be considered as a complex Gaussian process with zero mean. Meanwhile the receiving end of the interference signal is a white Gaussian noise with zero mean. Thus, based on the central limit theorem, the following approximate expression can be found:

$$
\mathrm{T}(\mathrm{y}) \sim\left\{\begin{array}{ll}
N\left(\sigma^{2}, \frac{2}{W} \sigma^{4}\right) & \text { for } H_{0} \\
N\left((1+\gamma) \sigma^{2}, \frac{2}{W}(1+2 \gamma) \sigma^{4}\right) & \text { for } H_{1}
\end{array},\right.
$$

In Eq.(3), $\gamma=\frac{\sigma_{p}^{2}}{\sigma^{2}}$ and $\gamma$ is the SNR under hypothesis $H_{1}$ and $\sigma_{p}^{2}$ is defined as the power of the PUs signal.

Based on Eq(3), the probabilityof detection $P_{d}$ and false alarm $P_{f}$ can be obtained by

$$
\begin{gathered}
P_{d}=P\left(T(y)>\lambda \mid H_{1}\right)=Q\left(\frac{\lambda-(1+\gamma) \sigma^{2}}{\sigma^{2} \sqrt{(1+2 \gamma) \frac{2}{W}}}\right), \\
P_{f}=P\left(T(y)>\lambda \mid H_{0}\right)=Q\left(\frac{\lambda-\sigma^{2}}{\sigma^{2} \sqrt{\frac{2}{W}}}\right),
\end{gathered}
$$

In (4) and (5), $\lambda$ is an assumed threshold value that will make the energy detection run in the optimal performance, and the symbol $\lambda$ needs to bederived and $Q(\cdot)$ represents the Q-Function which me.

There is not enough information for the model to define the probability of missed detection. In this paper, theprobability of missed detection is determined to be equal to the probability of the false alarm.In that case, the threshold value $\lambda$ can be derived, based on the following expression:

$$
1-P_{d}=P_{f}
$$

Substituting Eq.(4) and (5) into (6), the result is:

$$
\lambda=\sigma^{2}\left(1+\frac{\gamma}{1+\sqrt{1+2 \gamma}}\right) .
$$

\section{Ad Hoc Network}

\section{Introduce UDP.}

The User Datagram Protocol (UDP) designed by David P. Reed [9] is one of the important components of the Internet Protocol Suite. UDP does not provide any handshaking mechanism for guaranteeing packets reliability, packets ordering and data integrity. Time-sensitive and real-time applications like voice and video traffic use UDP because dropping packets is preferable instead of long delay of packets. UDP flow is consist of a set of packets with the same 5-tuple and its packet inter-arrival time does not exceed the fixed timeout 64s. [12] In this case, UDP is used to realize the transmission.

\section{UDP header.}

The following picture shows the UDP header, an important part for an equipment to transform. The header is used to identify the data packet.

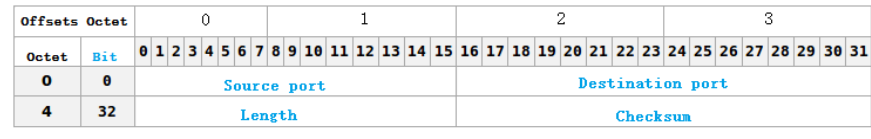

Fig. 2. UDP header 
In Fig.2, Source port denote the beginning port of UDP packet.Destination port denote the destination of the UDP packet.Lengthdenote the length of the UDP packet.Checksum denotes the Check of all data packet throughout the UDP.

\section{CBR.}

The Case-Based Reasoning (CBR) is a powerful and reflex natural. It rooted in early 1980s, is a major paradigm in automated reasoning and machine learning. [13] CBR is a method of solving problems, which is different from other artificial intelligence (AI) approaches. In particular, it uses the specific knowledge of concrete, previously experienced, problem situations. [14] Thus, It is useful to apply the CBR in our ad hoc Networks.

The Net work topology structure.

The topology structure shows how these three SUs work in the network. In Fig.3, SU0 and SU1 are used UDP/CBR to exchangeinformation

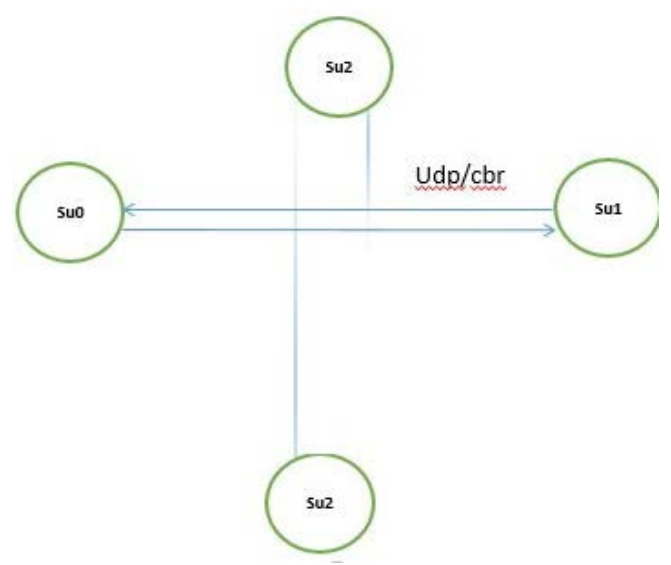

Fig. 3. The network Topology Structure: SU0 and SU1 are exchanging information by suing $\mathrm{UDP} / \mathrm{CBR}$

\section{Simulation}

Ns2 is a discrete event simulator targeted at networking research. Ns2 provides substantial support for simulation of UDP, routing, and multicast protocols over wireless networks

Algorithm of NS2.

This network need use the NS2 to simulate. Therefore, there are some major algorithm to show how the Network works.

a) Create channel and God:

set chan [new \$val(chan)]

set god [create-god \$val(nn)]

b) Load the god object with shortest hop information:

\$god set-dist 121

\$god set-dist 022

\$god set-dist 011

c) Su1 starts to move upward and then downward:

set god [God instance]

d) When the time is $200 \mathrm{~s}$, the su1 starting from $(500,500)$ to the $(500,900)$, it runs with $2.0 \mathrm{~m} / \mathrm{s}$ :

\$ns at 200.0 "\$node_(1) setdest $500.0900 .02 .0 "$

e) When the time is 500s, the su1 starting from $(500,500)$ to the $(500,900)$, it runs with $2.0 \mathrm{~m} / \mathrm{s}$ :

\$ns at 500.0 "\$node_(1) setdest 500.0 100.0 2.0"

f) Set the primary location of the $S U$

\$node(0) set X_250.0

\$node(0) set $Y_{-} 500.0$ 
\$node(0) set Z_ 0.0

\$node(1) set $X \_500.0$

\$node(1) set $Y_{-} 500.0$

\$node(1) set Z_ 0.0

\$node(2) set $X_{-} 750.0$

\$node(2) set $\mathrm{Y}_{-} 500.0$

\$node(2) set Z_ 0.0

g) Establish the CBR/UDP between the su0 and su2:

set udp_(0) [new Agent/UDP]

\$udp_(0) set fid_1

\$ns attach-agent \$node_(0) \$udp_(0)

set null_(0) [new Agent/Null]

\$ns attach-agent \$node_(2) \$null_(0)

set cbr_(0) [new Application/Traffic/CBR]

\$cbr_(0) set packetSize_ 100

\$cbr_(0) set interval_ 2.0

\$cbr_(0) set random_1

\$cbr_(0) set maxpkts_ 10000

\$cbr_(0) attach-agent \$udp_(0)

\$ns connect \$udp_(0) \$null_(0)

\$ns at 100.0 "\$cbr_(0) start"

h) Tell Sus when the simulation ends:

for $\{$ set i 0$\}\{\$ \mathrm{i}<\$ \mathrm{val}(\mathrm{nn})\}$ incr i $\}$ \}

\$ns initial_node_pos \$node_(\$i) 60

\}

for $\{$ set i 0$\}\{\$$ i $<\$$ val(nn) $\}$ incr i $\}\{$

\$ns at \$val(stop) "\$node_(\$i) reset";

\}

\section{Throughout.}

In a specified amount of time, the amount of data transferred from one place to another. Data transfer rates for disk drives and network are measured in terms of kilobits per second, megabits per second and etc.

This is followed by the eq.(8):

\section{Throughout $=$ Transfer size/ Transfer time(8)}

This model can use AWK to analyze the data in the 'Trace File', and use GNUPLOT to show the figure of throughout. Now, the following shows thealgorithm of the THROUGHOUT and its figure.

1) Algorithm of the Throughout

a) Give the new defination of each bit

event $=\$ 1$;

time $=\$ 2 ;$

node $=\$ 3$;

trace_type $=\$ 4$;

flag $=\$ 5$;

uid $=\$ 6$;

pkt_type $=\$ 7$;

pkt_size $=\$ 8$;

b) Calculate the through-out in different time:

for $(j=1 ; j<i ; j++)\{$

th=pkt_byte_sum[j]/(end_time[j]-start_time)*8/1000; 
2) The figure of throughout

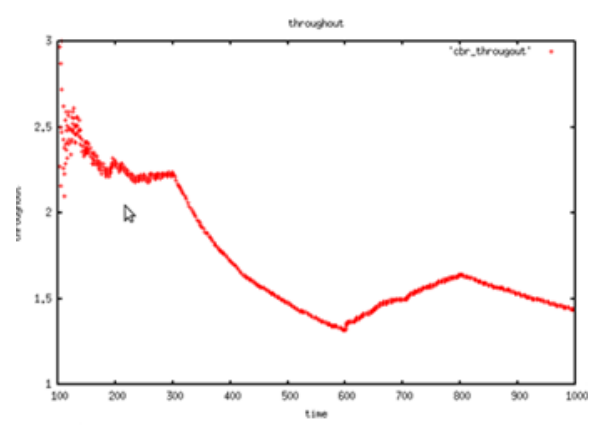

Fig. 4. Throughout of the network

In fig.4, the throughout is a little small, and during the movement of SU2, the throughout is becoming smaller and smaller.

\section{Packet loss Rate.}

Packet loss rate is the ratio of the amount of correctly received bytes at the destination port to the total amount of bytes transmitted at the source port.

This is followed by the eq. (9):

$$
\text { Packet Loss Rate }=\frac{\text { The number of sending Packets }}{\text { The number of Receiving packets }}(9)
$$

1) Algorithm of Packet Loss Rate

c) Give the new defination of each bit:

event $=\$ 1$;

time $=\$ 2 ;$

node $=\$ 3$;

trace_type $=\$ 4$;

flag $=\$ 5$;

uid $=\$ 6$;

pkt_type $=\$ 7$;

pkt_size $=\$ 8$

d) Calulate the number of the packets from source node:

sendsum++;

e) Calulate the received packets's number:

$$
\text { if(event=="s"\&\&pkt_type=="cbr"\&\&trace_type=="AGT") }
$$

recvsum++;

$$
\text { if(event=="r"\&\&pkt_type=="cbr"\&\&trace_type=="AGT") }
$$

f) Calulate the rate of packet-quiting

losssum=sendsum-recvsum;

loss_rate=losssum/sendsum;

2) Show the packet loss rate

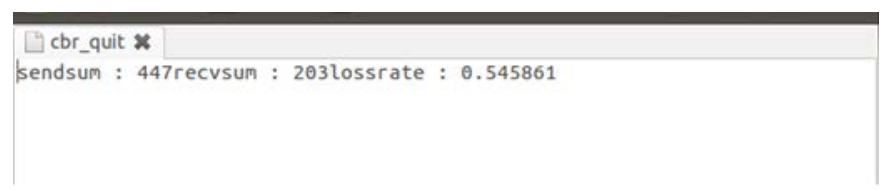

Fig. 5. Packet loss Rate of the network

In this figure, the Packet loss rate is $54 \%$. It is very large, which affects the communication between the SUs. 


\section{Delay.}

The time taken by the data packet to reach the intended destination, and here we considered Endto-Enddelay.

This is followed by the eq. (10):

$$
\sum_{j=1}^{n} \text { delay of the packet (10) }
$$

1) Algorithm of the Delay

g) Give the new defination of each bit

event $=\$ 1$;

time $=\$ 2 ;$

node $=\$ 3$;

trace_type $=\$ 4$;

flag $=\$ 5$;

uid $=\$ 6$;

pkt_type $=\$ 7$;

pkt_size = \$8;

h) Calculate the delay

If(event=="s"\&\&pkt_type=="cbr"\&\&uid > highest_uid ) \{

highest_uid = uid;

\}

If(event=="s"\&\&pkt_type=="cbr"\&\&uid==highest_uid\&\&trace_type=="AGT") s

tart_time[uid] = time;

\}

If(event=="r"\&\&pkt_type=="cbr"\&\&uid==highest_uid\&\&trace_type=="AGT" )\{

end_time[uid] = time;

\} if( event=="D")\{

end_time[uid] =-1;

\}

\}

2) the Figure of Delay

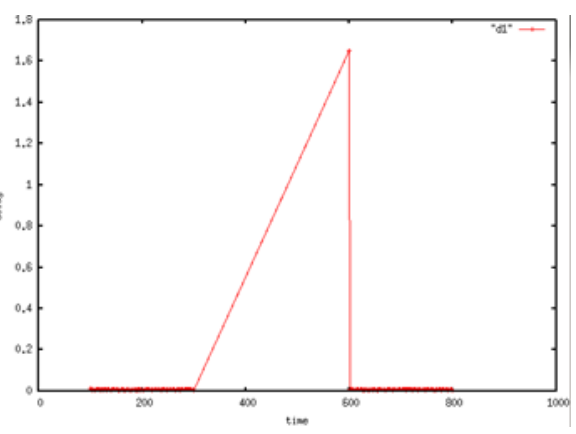

Fig. 6. Delay of the network

In fig.6, the Delay is very large. This must influence the ad hoc networks runs, and it must cause the congestion.

\section{Conclusion}

In this paper, UDP/CBR is used for SUs' information exchanges in a CR. Energy detection technology with an optimal thresholdis employed to find the idle spectrum resource. These resources will be utilized to build the Ad Hoc Network with the assistance of the computer network protocol. 
The simulation on the NS2 is also successfully accomplished.After finishing the simulation of the Ad Hoc network, it indicatesthat the proposed ideain the paper works well. However, there are some problems remains, for example, delay and the through-out is not so good, which is the future work.

\section{References}

[1] C. Bazelon, " Licensed or unlicensed: The economic considerations in incremental spectrum allocations,"IEEE Communications Magazine, vol.11, no.3, pp.110-116, 2009

[2] S. Haykin, "Cognitive radio: brain-empowered wireless communications,"IEEE Journal on Selected Areas in Communications, vol.23, no.2, pp.201-220, 2005

[3] J. Mitola, "Software radio architecture: a mathematical perspective,", IEEE Journal on Selected Areas in Communications, vol.17, no.4, pp.514-528, 1999

[4] R. Etkin, A. Parekh and D. Tse, "Spectrum sharing for unlicensed bands," IEEE Journal on Selected Areas in Communications, vol.25, no.3, pp.517-528, 2007

[5] T. Yucek, and H. Arslan, "A survey of spectrum sensing algorithms for cognitive radio applications," IEEE Communications Surveys \& Tutorials, vol.11, no.1, pp.116-130, 2009

[6] A. Ghasemi and E.S. Sousa, "Optimization of spectrum sensing for opportunistic spectrum access in cognitive radio networks," IEEE Consumer Communications and Networking Conference, pp.1022-1026, 2007

[7] H. Urkowitz, "Energy detection of unknown deterministic signals," Proceedings of the IEEE,vol.55, no.4, pp.523-531, 1967

[8] Z. Li,, F. R. Yu and M. Huang, "A distributed consensus-based cooperativespectrum-sensing scheme in cognitive radios," IEEE Transactions on Vehicular Technology, vol.59, no.1,pp.383393, 2010

[9] M.A. Azad, R. Mahmood and T. Mehmood, "A comparative analysis of DCCP variants (CCID2, CCID3), TCP and UDP for MPEG4 video applications," IEEE International Conference onInformation and Communication Technologies, pp.40-45,2009

[10] K. Iida, T. Takine, H. Sunahara andY. Oie, "Delay analysis for CBR traffic in static-priority scheduling: single-node and homogeneous CBR traffic case." Voice, Video, and Data Communications. International Society for Optics and Photonics, vol.2, pp.1256-1263, 1997

[11] P.A.A. Assuncao andM. Ghanbari, "Buffer analysis and control in CBR video transcoding," IEEE Circuits and Systems for Video Technology, vol.10, no.1, pp.83-92, 2000

[12] J. Cai, Z. Zhang, P. Zhang and X. Song "An adaptive timeout strategy for profiling UDP flows," IEEE Networking and Computing (ICNC) Conference, pp.44-48, 2010

[13] N. Dendani-hadiby, M.T Khadir, "A fault diagnosis application based on a combination casebased reasoning and ontology approach." International Journal of Knowledge-based and Intelligent Engineering Systems, vol.17, no.4, pp.305-317, 2013

[14] P. Zhou, W. Yin and J. Zhao, "Research on case retention strategy for industrial case-based reasoning (CBR) system: A practical case study," IEEE Computer Science and Information Processing (CSIP) Conference, pp.376-380,2012 